\title{
Title: Network stress test reveals novel drug potentiators in
}

\section{Mycobacterium tuberculosis}

Authors: Shuyi Ma ${ }^{1,2}$, Robert Morrison ${ }^{1}$, Samuel J. Hobbs ${ }^{1}$, Jessica Farrow-Johnson ${ }^{1}$,

Tige R. Rustad ${ }^{1,2}$, David R. Sherman ${ }^{1,2 *}$

Affiliations:

${ }^{1}$ Center for Infectious Disease Research, formerly Seattle Biomedical Research Institute, Seattle, Washington, 98109, USA.

${ }^{2}$ Interdisciplinary Program of Pathobiology, Department of Global Health, University of Washington, Seattle, Washington 98195, USA.

*Correspondence to: David R. Sherman (email: david.sherman@cidresearch.org).

\section{Main Text:}

Deciphering molecular stress response is highly relevant to studies of microbes such as Mycobacterium tuberculosis (MTB), the causative pathogen of tuberculosis (TB) which adaptations and how they are regulated can reveal new biology, including unexplored drug targets and treatment-enhancing strategies. Here, we present a novel networkbased genetic screening approach: the Transcriptional Regulator Induced Phenotype 
41 of multiple genes.

adaptations to the first-line drug isoniazid $(\mathrm{INH})$. We found regulators that alter $\mathrm{INH}$ susceptibility when induced, several of which could not be identified by standard gene disruption approaches. We then focused on a specific regulator, mce3R, which potentiated INH when induced. We compared mce3R-regulated genes with the baseline INH transcriptional response and implicated the gene Rv1469 as a putative INH effector. Phenotyping a disruption mutant strain then demonstrated a previously unknown role for Rv1469 in INH susceptibility. Integrating TRIP screening with network data can uncover sophisticated molecular response programs.

Stress response in bacteria has been studied both through targeted characterization of individual genes and by systems-level screens. In-depth characterization of specific genes is necessary to elucidate molecular details, but these efforts are time-and resource-intensive. Systems-level studies often rely on high-throughput phenotypic profiling of transposon mutagenesis libraries (e.g. Tn-seq, TraSH, HITS, IN-seq, TraDIS) ${ }^{5-12}$. These approaches assess differential viability among pools of transposonmediated gene disruption mutants, enabling unbiased screening for putative candidate genes. While powerful, these methods pose some limitations: 1) they cannot identify genes whose upregulation generates a phenotype, 2) essential genes cannot be profiled, and 3) they miss emergent phenotypes that arise only from coordinated actions 
To address these limitations, we developed a strategy that quantifies growth associated with individually inducing each MTB transcription factor (TF). This Iranscriptional Regulator Induced Phenotype (TRIP) screen exploits a library of 207 TF-induction (TFI) strains, representing $97 \%$ of annotated MTB regulators, each transformed with a plasmid carrying an MTB TF under control of a mycobacterial chemically-inducible promoter (see Methods for details) (Figure 1). Each strain is engineered for conditional induction of a single MTB TF and expression of its associated regulon-the set of genes whose expression changes when a given TF is induced ${ }^{13-15}$. ChIP-seq and expression profiling of these TFI strains under in vitro logphase conditions revealed a baseline network of transcriptional impacts and DNA binding

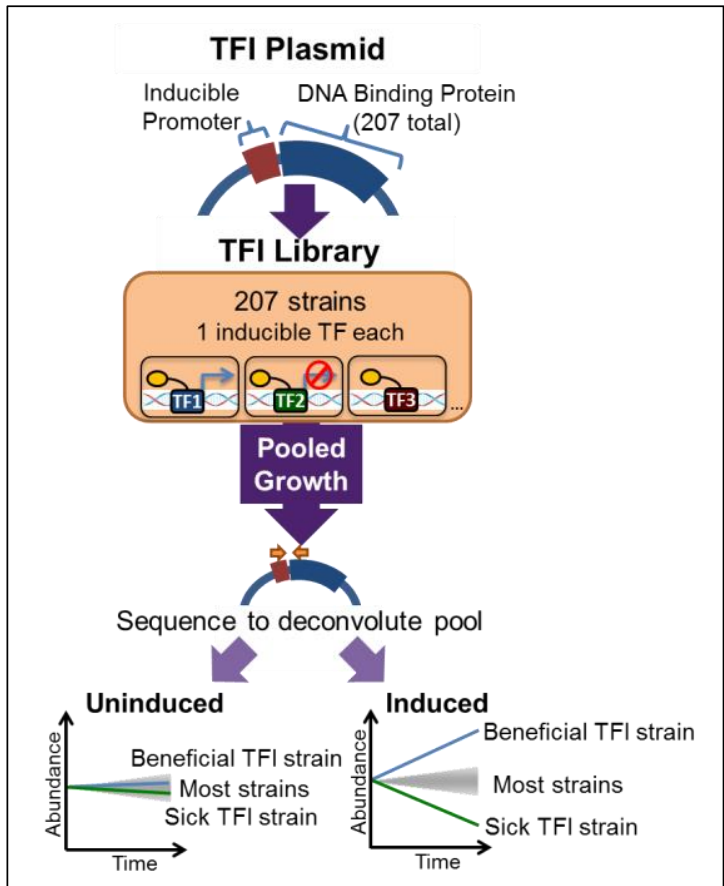

Figure 1. Schematic of TRIP screen. interactions triggered by each TF ${ }^{13-15}$.

Here, we pool the TFI strain library for simultaneous measurement of strain growth (Figure 1). The pool is exposed to a stress condition either with or without TF induction. The proportion of each TFI strain in the pool is quantified by next-generation sequencing of a DNA segment unique to each strain (see Methods for details). Sampling the pooled culture over time generates simultaneous abundance curves for each TFI strain. The fold abundance change of each strain when induced compared to uninduced identifies regulons with altered growth or survival. 
TRIP screens offer several powerful advantages: 1) emergent, network phenotypes are accessible, since regulons generally include multiple genes selected for co-regulation by evolution; 2) revealed phenotypes can be deconstructed with the existing baseline regulatory network; 3) TF expression is chemically triggered, enabling context-specific interrogation of perturbations; and 4) essential regulators and effector genes can be assessed. Moreover, TRIP is broadly extensible—the pipeline can be harnessed to interrogate MTB phenotypes under numerous stress conditions, and the fundamental strategy can be adapted to investigate other species. Thus, TRIP is a highly complementary strategy to transposon-based approaches.

We first applied TRIP to MTB log-phase growth in vitro to characterize network perturbations that alter baseline fitness. Figure 2A visualizes abundance fold change of each TFI strain when induced in these conditions (see tab 1 of Table S1 for detailed results). Most TFI strains showed no significant difference in abundance upon induction. Twenty-two TFI strains (10.6\%; below dotted line at -0.5) exhibited substantial growth defects upon TF induction. (see Methods for details).

Growth-defective strains are enriched in TFs that activate genes associated with starvation responses (Table S2). Such strains are also enriched for TFs that repress essential genes ( $p<10^{-6}$, hypergeometric test), although two defect-inducing TFIs (Rv3765c and Rv1255c) do not repress any essential genes, and 20 TFIs with no discernable growth phenotype do repress essential genes ${ }^{7}$. There is no significant correlation between the number of repressed essential genes and extent of growth defect incurred by the TFI strain. 
To validate differences in relative abundance detected by TRIP, we compared the TRIP output with the growth of each individual TFI strain over a one-week time course with and without TF induction. Of the 22 TFI strains with strong growth defects in the TRIP assay, 20 also had strong growth defects when cultured individually. This validation indicates that 1) phenotypes detected by TRIP reflect growth observed in monoculture, and 2) significant growth defect upon TF induction is an uncommon and highly TFspecific effect.

Once baseline MTB network phenotypes were established, we applied TRIP to study response to INH. We exposed TFI pools to a dose of INH where the bulk population grew suboptimally (19\% of untreated, see Figure S1), enabling us to identify TFIs with reduced and improved viability compared to the population average in a single experiment. Figure 2D shows abundance of each TFI strain exposed to drug in the induced condition relative to uninduced. TFI strains with a growth phenotype specific to INH partition into three groups: A) TFIs that convey growth advantage in INH but no change in when untreated (7 strains, purple box); B) TFIs that convey growth defect in INH but no change when untreated (4 strains, blue box); and C) TFls that convey growth defect in both INH and untreated conditions (9 strains; light blue box). The genes whose promoters are bound by TFs in group A are enriched for transporter functions Table S2). In contrast, genes activated by TFs in groups B and C are significantly enriched for several stress response, homeostasis, and transport processes, and genes repressed by these TFs are significantly enriched for NADH dehydrogenase (ubiquinone) activity and DNA replication initiation (Table S2). Out of the 20 TFs associated with INH phenotypes by TRIP, two of them were reported by Xu and 
110 colleagues ${ }^{16}$ to alter MTB fitness significantly during INH treatment by Tn-seq (Table

111 S2). The regulons of TFs in all three groups were enriched for genes reported to alter

112 INH fitness significantly by Tn-seq (Table S3) ${ }^{16}$. Notably, two of the four TFs in group B

113 solely activate genes when induced (Rv0330c and Rv2282c), so the association

114 between members of these regulons and INH fitness could not have been detected by

115 gene disruption-based assays.

116 The TFI conveying the greatest survival advantage during INH exposure is Rv1909c/

117 furA. This TF represses expression of $k a t G(R v 1908 c)$, which encodes the catalase-

118 peroxidase enzyme that converts the INH prodrug into its active form ${ }^{17,18}$. Inducing furA

119 in the presence of INH is known to restore nearly uninhibited growth $14,17,19$.

120 We next sought to investigate regulons that represented potential novel therapeutic

121 targets. The TF conveying the greatest growth defect upon induction in INH is Rv1963c

122 (mce3R), a TetR-like regulator. mce3R has previously been linked to the expression of

123 genes that mediate $\beta$-oxidation of fatty acids and lipid transport ${ }^{20-22}$ and had no

124 previous connection to INH. 
To validate its hypersusceptibility, we tested viability of the mce $3 R$ induction strain in
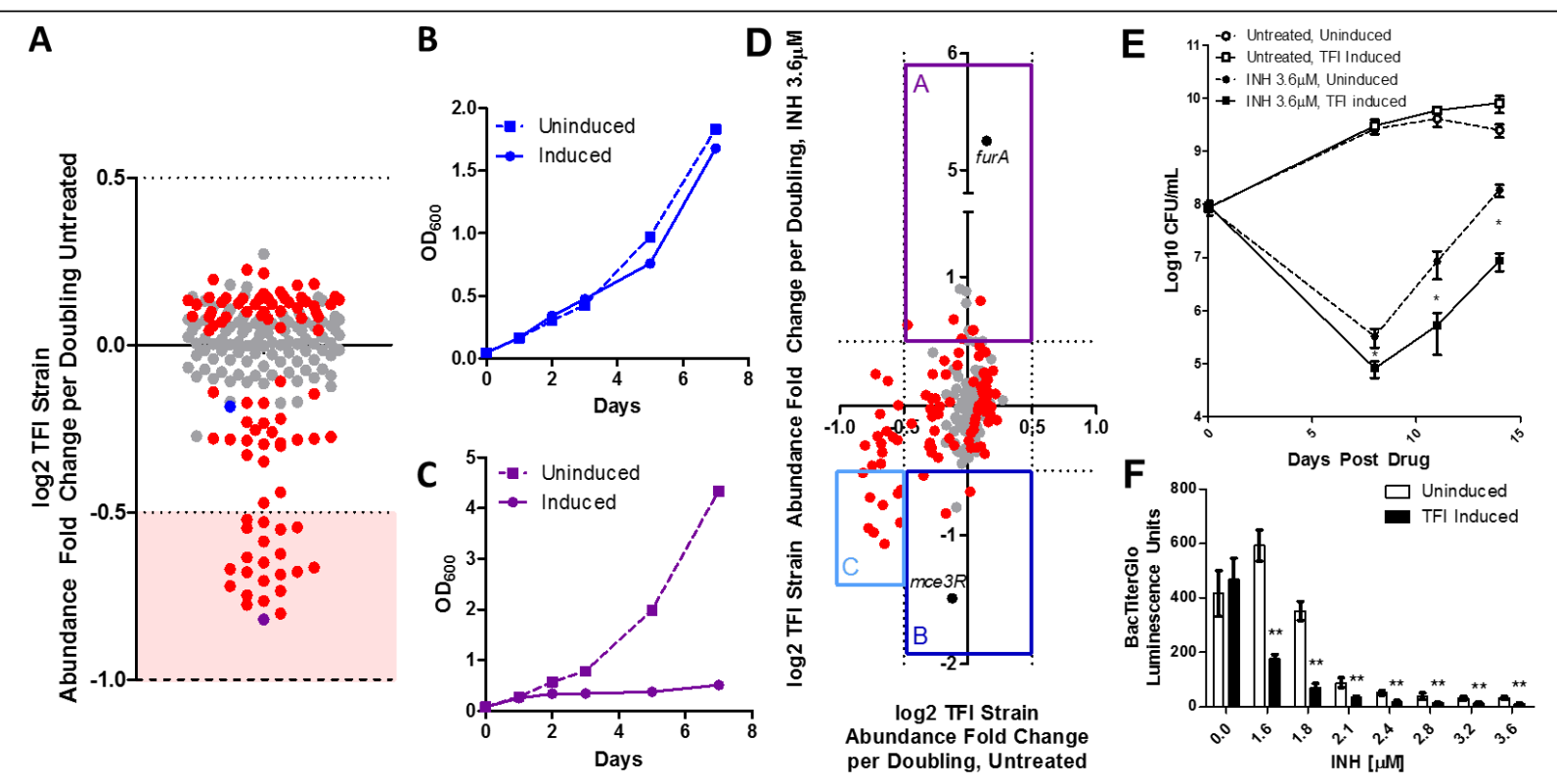

Figure 2. Regulon-mediated growth responses. (A) Log-phase TRIP results. Dots represent abundance change of TFI strains induced vs. uninduced, normalized by the estimated number of pool doublings. Red dots indicate $z$-score $>1$, from three replicates. Dotted lines specify \pm 2 standard deviations, and dashed line denotes detection limit, signifying no growth. Shaded area represents strains with strong defects. Monoculture growth curves of two strains (blue, purple dots) are shown in (B) and (C) respectively, with induction (solid) or without (dashed). (D) TRIP results with INH (y-axis) vs. no drug (x-axis). Since INH can be bactericidal, some strains showed abundance changes $<-1$. Boxes demarcate strains with altered INH survival. Black dots represent strains: Rv1909c (furATFI) and Rv1963c (mce3RTFI). (E) mce3RTFI colony forming units (CFU)/mL over 14 days in INH (black) vs. no drug (white) with induction (solid) or without (dashed). Error bars show standard deviation from three replicates. (F) mce3RTFI metabolic viability after 7 days INH with induction (black) or without (white), measured by luminescence (see Methods). Error bars show standard deviation from four replicates. * and ** indicate significant differences between induction states $(p<0.05$ and $p<0.001$, Student's t-test, respectively). 
135 Hypersusceptibility could stem from some TFI-mediated countering of the MTB

136 adaptation to INH. To investigate this hypothesis, we compared the mce3R induction

137 regulon determined in our basal transcriptional network with the genes differentially

138 expressed when H37Rv is exposed to INH ${ }^{14,23,24}$. Rv1469 is one of two genes

139 repressed by mce3R (Figure $3 \mathbf{A}$, see Figure S3 for full set), and Rv1469 is normally

140 upregulated in response to INH in broth culture and under macrophage infection

141 conditions ${ }^{23,24}$. After ruling out the other gene (see Table S4 and Table S5 for details),

142 we hypothesized that Rv1469 induction might be important for temporary MTB

143 adaptation to INH. If so, reducing Rv1469 expression might contribute to INH

144 hypersusceptiblity independent of expression changes in mce3R.

145 To test whether Rv1469 could influence INH susceptibility, we obtained a transposon

146 mutant that disrupted Rv1469 (see Methods for details). We compared kill curves for

147 this mutant (Rv1469::Himar1) vs. the parent strain CDC1551 when exposed to INH

148 (Figure 3B). As predicted, survival was reduced in Rv1469::Himar1 relative to

149 CDC1551 following INH treatment (42-fold difference after 7 days, $p<10^{-4}$, Student's t-

150 test), with no significant growth difference in absence of drug. Intriguingly, Rv1469 was

151 not associated with significantly altered INH susceptibility in the Tn-seq screen by Xu

152 and colleagues ${ }^{16}$. 
Rv1469 encodes a membrane protein ${ }^{25}$ annotated as the MTB homolog of CtpD, a member of the metal cation-transporting P1B4-ATPase subgroup, which is essential for MTB survival in the host ${ }^{6,26,27}$. CtpD is a high-affinity $\mathrm{Fe}^{2+}$ exporter needed for overcoming redox stress and adapting to the host environment ${ }^{26,28}$. Given that KatGmediated catalysis is iron-dependent, $\mathrm{Fe}^{2+}$ accumulation resulting from loss of $\operatorname{ctp} D$

\section{A}

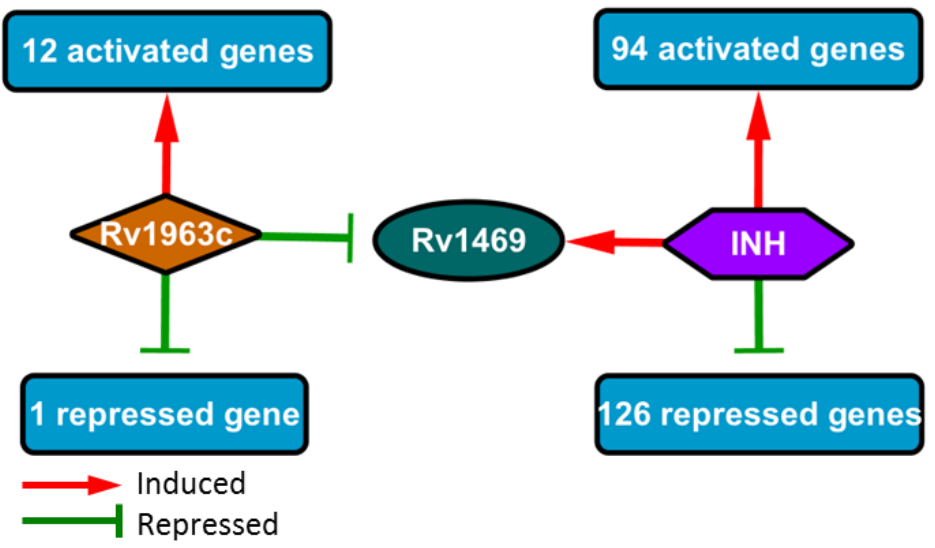

B

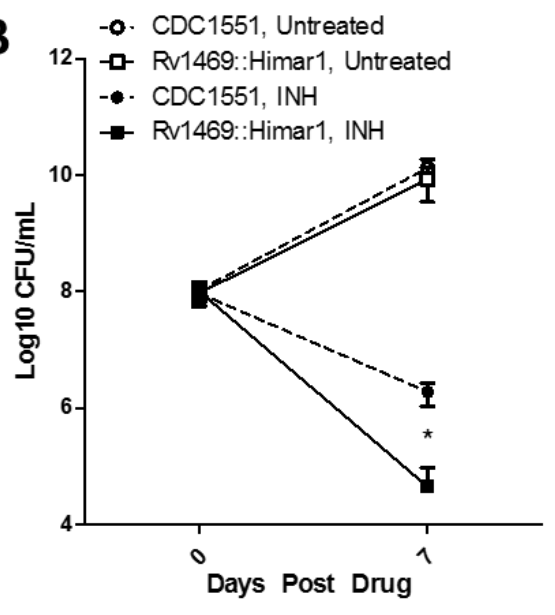

Figure 3. $m c e 3 R$ regulon reveals novel effector of INH susceptibility. (A) Network representation of overlap between $m c e 3 R$ regulon and genes differentially expressed in wildtype response to INH exposure. Red arrows indicate genes activated at least 2-fold, and green lines indicate genes repressed at least 2-fold. (B) $\mathrm{CFU} / \mathrm{mL}$ over 7 days of Rv1469::Himar1 transposon disruption strain (solid) compared to the wildtype strain, CDC1551 (dashed). Both strains were exposed to $3.6 \mu \mathrm{M}$ INH (black) vs. no drug (white). Error bars indicate standard deviation from three replicates. The * indicates significant difference between the mutant and wildtype strains. 
166 First, by targeting regulons, TRIP harnesses nature's levers to modulate responses-

167 tuning gene sets that evolved to change coordinately-and uncovers emergent

168 phenotypes that depend on synchronized actions of multiple genes. For example, two

169 TFls that slowed growth under log-phase conditions (Rv3765c and Rv1255c) do not repress any essential genes, suggesting that epistatic mechanisms may underlie these

171 growth defects. Epistasis may also explain how TRIP successfully identified mce3R as

172 a key regulatory potentiator of INH activity, whereas one of its target effector genes,

$173 c t p D$, was not identified in a recent $\mathrm{Tn}$-seq screen for $\mathrm{INH}$-associated genes. In addition,

174 TRIP samples network states that are distinct from those resulting from TF disruption.

175 For example, mce3R was previously reported to regulate the mce3 operon genes based on studies of a deletion mutant ${ }^{20,21}$. However, the transcriptional impact of inducing mce3R does not include the mce3 operon (see tab 3 of Table S2 for full regulon, based

178 on data from ${ }^{14}$ ), suggesting that mce3R participates in complex regulatory circuits.

179 Combining gene disruption studies with TRIP screens and network analysis and could

180 facilitate untangling these complex, nonlinear phenotypes. Finally, unlike gene-

181 disruption assays, TRIP can identify fitness phenotypes associated with gene upregulation, such as in the cases of the INH hypersusceptibility-inducing TFs Rv0330c

183 and Rv2282c, both of which exclusively activate genes when induced.

184 In the current study, we combined TRIP results with network analysis to identify novel

185 genes that altered MTB response to the frontline drug INH. However, TRIP can

186 interrogate network mediators of growth and survival under any condition from which

187 microbes can be recovered, and TRIP requires tracking a substantially reduced set of

188 mutants compared to Tn-seq, rendering it technically tractable. By integrating with 
network insight, TRIP will lend novel insights into emergent mechanisms underlying condition-specific growth phenotypes for MTB, and the strategy can be generalized for other organisms as well.

\section{Methods:}

\section{Strains and expression vectors.}

The individual strains comprising the MTB Transcription Factor Induction (TFI) Library were generated previously ${ }^{14}$. Briefly, 207 MTB DNA binding genes were cloned into a tagged, inducible vector that placed the TF under control of a tetracycline-inducible promoter and added a C-terminal FLAG epitope tag ${ }^{14,31-34}$. The constructs were then individually transformed into MTB H37Rv using standard methods. Individual TFI strains are available from the BEI strain repository at ATCC $(35, N R-46512)$. The TFI library was generated by combining equal proportions of each strain into a common pool.

The Rv1469 transposon strain was obtained through BEI Resources, NIAID, NIH: MTB: Strain CDC1551, Transposon Mutant 1738 (MT1515, Rv1469), NR-18218 ${ }^{35}$. The transposon insertion is located at base 671 in the 1974 base-pair long gene ${ }^{35}$.

$\underline{\text { Culture. }}$

Bacteria were cultured at $37^{\circ} \mathrm{C}$ under aerobic conditions with constant agitation. For the experiments involving TFI strains, the strains were cultured in Middlebrook $7 \mathrm{H} 9$ with the ADC supplement (Difco), $0.05 \%$ Tween 80 , and $50 \mu \mathrm{g} / \mathrm{mL}$ hygromycin B to maintain the plasmids. 
210 For the TRIP experiments, growth of the pooled TFI library was monitored by OD600. At

211 an OD600 of 0.1 , expression of the pooled TFI library was induced with

212 anhydrotetracycline (ATc, $100 \mathrm{ng} / \mathrm{mL})$, and the cultures were grown for 7 days

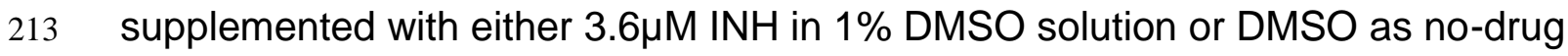

214 control. The cultures were sampled at Day 0 and Day 7 of the experiment for DNA

215 isolation and subsequent sequencing.

216 For individual TFI strain time course experiments, each strain was cultured under the

217 same media conditions as described for the pooled TFI library. When cultures reached

218 OD600 0.1, TFI strain induction and drug exposure proceeded as described for the

219 pooled TFI library. The individual strain cultures were monitored for up to 14 days, with

220 viability under the different drug and induction conditions assayed by plating on

221 Middlebrook 7H10 solid media plates and assessing colony forming units using

222 standard methods.

223 The Rv1469 transposon mutant strain was cultured in Middlebrook 7H9 with ADC

224 supplement (Difco), 0.05\% Tween80, and $30 \mu \mathrm{g} / \mathrm{mL}$ kanamycin to maintain the

225 transposon insertion. Growth and survival of Rv1469 mutant was compared against the

226 parent MTB CDC1551 strain. When cultures reached an OD600 of 0.1, drug exposure

227 proceeded as described for the pooled TFI library. The individual strain cultures were

228 monitored for up to 14 days, with viability under the different drug and induction

229 conditions assayed by plating on Middlebrook $7 \mathrm{H} 10$ solid media plates and assessing

230 colony forming units using standard methods. 
232 Strains were grown to log phase (OD600 0.3), diluted to a final OD600 of 0.005 , and dispensed into 96-well flat-bottom plates (Corning, Acton, MA) at a final volume of $200 \mu \mathrm{L}$, containing $1 \% \mathrm{DMSO}$ and varying concentrations of $\mathrm{INH}$ in the different wells.

235 On each plate, control wells for each of the strains studied were included, containing no

236 drug and $1 \%$ DMSO vehicle, to measure viability in the absence of INH exposure.

237 Plates were incubated at $37^{\circ} \mathrm{C}$ for 7 days. Cellular viability was assayed on Day 7 by

238 adding $20 \mu \mathrm{L}$ of culture from each well to $20 \mu \mathrm{L}$ of BacTiter-Glo Microbial Cell Viability

239 Assay Reagent (Promega, Madison, WI), incubating at room temperature protected

240 from direct light for 20 minutes, and reading luminescence intensity using a FluoStar

241 Omega plate reader (BMG Lab Tech, Cary, NC).

$242 \quad$ DNA isolation and sequencing

243 Cell pellets collected from each sample were resuspended in TE buffer, $\mathrm{pH}$ 8.0,

244 transferred to a tube containing Lysing Matrix B (QBiogene, Inc.), and vigorously 245 shaken three times at $6 \mathrm{~m} / \mathrm{s}$ for 30 seconds per cycle in a Bead Ruptor 24 homogenizer

246 (Omni International, Kennesaw, GA), with a 30-second pause between each cycle. The

247 mixture was centrifuged at maximum speed for one minute, and DNA was extracted

248 from the supernatant using the MagJet Genomic DNA Kit (Thermo Fisher), according to

249 the manufacturer's instructions for manual genomic DNA purification.

250 PCR pre-amplification of DNA barcodes unique to each TFI strain was performed. The

251 products of this reaction were prepared for Illumina sequencing using the NEBNext

252 Ultra DNA Library Prep Kit for Illumina (New England Biolabs, Ipswich, MA) according to

253 manufacturer's instructions, and using the AMPure XP reagent (Agencourt Bioscience

254 Corporation, Beverly, MA) for size selection and cleanup of adaptor-ligated DNA. We 
used the NEBNext Multiplex Oligos for Illumina (Dual Index Primers Set 1) to barcode the DNA libraries associated with each replicate and enable multiplexing of 96 libraries per sequencing run. The prepared libraries were quantified using the Kapa qPCR quantification kit, and were sequenced at the University of Washington Northwest Genomics Center with the Illumina NextSeq 500 Mid Output v2 Kit (Illumina Inc, San Diego, CA). The sequencing generated an average of 1.5 million 75 base-pair pairedend raw read counts per library.

\section{$\underline{\text { Sequencing read alignment and TFI strain abundance deconvolution }}$}

Read alignment was carried out using a custom processing pipeline that harnesses the Bowtie 2 utilities ${ }^{36,37}$, which is available at

\section{https://github.com/DavidRShermanLab/TRIPscreen,}

https://github.com/sturkarslan/DuffyNGS, and https://github.com/sturkarslan/DuffyTools.

A custom Bowtie 2 target index was constructed from: the CDS sequences of all H37Rv genes; the inducible TFI anchor plasmid sequence; and the complete sequence of the empty plasmid as a negative control. The two mate-pair FASTQ datasets for each sample were separately mapped as unpaired reads using Bowtie 2's local alignment mode. After both mate end datasets were aligned separately, the alignment results were combined to give a pair of gene/plasmid alignments for each raw read. Only raw read pairs having one alignment to the anchor plasmid and the other to a gene with an existing "Rv" code were kept as valid reads. Read pairs that mapped to "Rv" code genes on both ends, or pairs that failed to align were discarded. On average, each sample had $99.9 \%$ valid anchor/gene reads, which is comparable to typical RNA-seq and WGS alignment results. Libraries that generated fewer than 10,000 valid read pairs 
were excluded from further analysis. Valid reads were then tallied for all "Rv" code genes reported as raw abundance measures. Read counts for each TFI were then normalized as $\log 2$ reads per million (RPM) values. Higher RPM values indicated that the corresponding TFI strain had greater relative abundance in the pooled culture. The average log2 RPM values across TFIs were 11.7 \pm 3.2 . TFIs with low abundance levels on day 0 of each experiment ( $\log 2 \mathrm{RPM}<5)$ were excluded from subsequent analysis (10 TFI strains, $4.8 \%)$.

To assess the effect of induction on TFI strain relative abundance, the log2 fold-change RPM values were calculated for the TFI-induced condition relative to un-induced. These values were further normalized by the number of doublings of the pooled library estimated from the change in OD600 over the course of the experiment. Positive fold change RPM values indicated that TFI induction conveyed a growth benefit, whereas negative fold change RPM values indicated that the TFI induction conveyed a growth defect under the conditions assayed. TFI strains that exhibited a log2 fold-change per doubling greater than 0.5 with z-score greater that 1 were deemed to have a significant growth phenotype under the condition assayed. The code for this processing is available at https://github.com/DavidRShermanLab/TRIPscreen.

\section{$\underline{\text { Statistics }}$}

Unless indicated, experiments were performed three times, and the mean and standard deviation from biological replicates of representative experiments are reported. Statistical differences between means were evaluated by two-tailed Student's t-tests, statistically significant correlation was evaluated by calculating a Pearson correlation coefficient and comparing against a Student's t distribution, and statistical enrichment 
301 was evaluated by hypergeometric test, unless otherwise noted. The significance cutoff

302 was set at $p<0.05$, unless otherwise indicated.

303 Gene Ontology Enrichment Analysis

304 The gene ontology (GO) term annotations for genes comprising the regulons of the TFs

305 under analysis were taken from ${ }^{38}$ and evaluated for statistical enrichment against the

306 GO annotations for the entire gene set of the MTB strain H37Rv using the

307 hypergeometric test and further subjected to a Bonferroni correction for multiple

308 hypothesis testing, with the number of independent tests estimated as the number of

309 GO terms associated to at least 2 genes in the H37Rv reference gene set (analogous to

310 method used by ${ }^{39}$ ). We further filtered the enriched GO terms to only those featured in

311 the regulons for 2 more of the TFs under analysis.

312 Data Availability

313 The data reported in the paper are available in the Supplementary Materials. The raw

314 .fastq sequence data files are deposited in the Sequence Read Archive database

315 [accession number pending]. The code required to process the sequenced reads are

316 available at: https://github.com/sturkarslan/DuffyNGS,

317 https://github.com/sturkarslan/DuffyTools, and

318 https://github.com/DavidRShermanLab/TRIPscreen. 
3231 WHO. Global Tuberculosis Report 2016. (2016).

3242 Warner, D. F. \& Mizrahi, V. Tuberculosis chemotherapy: the influence of bacillary stress

325 and damage response pathways on drug efficacy. Clin Microbiol Rev 19, 558-570,

326 doi:10.1128/CMR.00060-05 (2006).

3273 Aldridge, B. B., Keren, I. \& Fortune, S. M. The Spectrum of Drug Susceptibility in

328 Mycobacteria. Microbiol Spectr 2, doi:10.1128/microbiolspec.MGM2-0031-2013 (2014).

3294 Wallis, R. S. et al. Drug tolerance in Mycobacterium tuberculosis. Antimicrob Agents

$330 \quad$ Chemother 43, 2600-2606 (1999).

3315 Sassetti, C. M., Boyd, D. H. \& Rubin, E. J. Comprehensive identification of conditionally

332 essential genes in mycobacteria. Proc Natl Acad Sci U S A 98, 12712-12717,

333 doi:10.1073/pnas.231275498 (2001).

3346 Sassetti, C. M. \& Rubin, E. J. Genetic requirements for mycobacterial survival during

335 infection. Proc Natl Acad Sci U S A 100, 12989-12994, doi:10.1073/pnas.2134250100

$336 \quad(2003)$.

3377 Griffin, J. E. et al. High-resolution phenotypic profiling defines genes essential for

338 mycobacterial growth and cholesterol catabolism. PLoS Pathog 7, e1002251,

339 doi:10.1371/journal.ppat.1002251

340 PPATHOGENS-D-11-00689 [pii] (2011).

3418 Langridge, G. C. et al. Simultaneous assay of every Salmonella Typhi gene using one 342 million transposon mutants. Genome Res 19, 2308-2316, doi:10.1101/gr.097097.109 343 (2009). 
3449 Gallagher, L. A., Shendure, J. \& Manoil, C. Genome-scale identification of resistance functions in Pseudomonas aeruginosa using Tn-seq. MBio 2, e00315-00310, doi:10.1128/mBio.00315-10 (2011).

$347 \quad 10 \quad$ van Opijnen, T., Bodi, K. L. \& Camilli, A. Tn-seq: high-throughput parallel sequencing

11 Goodman, A. L. et al. Identifying genetic determinants needed to establish a human gut symbiont in its habitat. Cell Host Microbe 6, 279-289, doi:10.1016/j.chom.2009.08.003

12 Gawronski, J. D., Wong, S. M., Giannoukos, G., Ward, D. V. \& Akerley, B. J. Tracking insertion mutants within libraries by deep sequencing and a genome-wide screen for Haemophilus genes required in the lung. Proc Natl Acad Sci U S A 106, 16422-16427,

35713 Minch, K. J. et al. The DNA-binding network of Mycobacterium tuberculosis. Nat

14 Rustad, T. R. et al. Mapping and manipulating the Mycobacterium tuberculosis Commun 6, 5829, doi:10.1038/ncomms6829 (2015). transcriptome using a transcription factor overexpression-derived regulatory network. Genome Biol 15, 502, doi:10.1186/PREACCEPT-1701638048134699 (2014).

15 Galagan, J. E. et al. The Mycobacterium tuberculosis regulatory network and hypoxia. Nature 499, 178-183, doi:nature12337 [pii] 
$36516 \mathrm{Xu}, \mathrm{W}$. et al. Chemical Genetic Interaction Profiling Reveals Determinants of Intrinsic Antibiotic Resistance in Mycobacterium tuberculosis. Antimicrob Agents Chemother 61, doi:10.1128/AAC.01334-17 (2017).

36817 Pym, A. S. et al. Regulation of catalase-peroxidase (KatG) expression, isoniazid sensitivity and virulence by furA of Mycobacterium tuberculosis. Mol Microbiol 40, 879of catalase-peroxidase gene katG. Mol Microbiol 39, 1174-1185 (2001). doi:10.1038/358591a0 (1992). Mycobacterium tuberculosis. Microbiology 148, 2997-3006, doi:10.1099/00221287-14810-2997 (2002). isoniazid resistance of Mycobacterium tuberculosis. Nature 358, 591-593,

Santangelo, M. P. et al. Study of the role of Mce3R on the transcription of mce genes of Mycobacterium tuberculosis. BMC microbiology 8, 38, doi:10.1186/1471-2180-8-38 (2008).

de la Paz Santangelo, M. et al. Mce3R, a TetR-type transcriptional repressor, controls the expression of a regulon involved in lipid metabolism in Mycobacterium tuberculosis. Microbiology 155, 2245-2255, doi:10.1099/mic.0.027086-0 (2009).

23 Liu, Y. et al. Immune activation of the host cell induces drug tolerance in Mycobacterium tuberculosis both in vitro and in vivo. $J$ Exp Med 213, 809-825, doi:10.1084/jem.20151248 (2016). 
24 Ma, S. et al. Integrated Modeling of Gene Regulatory and Metabolic Networks in Mycobacterium tuberculosis. PLoS Comput Biol 11, e1004543, doi:10.1371/journal.pcbi.1004543 (2015).

25 Mawuenyega, K. G. et al. Mycobacterium tuberculosis functional network analysis by global subcellular protein profiling. Mol Biol Cell 16, 396-404, doi:10.1091/mbc.E04-040329 (2005).

26 Raimunda, D., Long, J. E., Padilla-Benavides, T., Sassetti, C. M. \& Arguello, J. M. Differential roles for the $\mathrm{Co}(2+) / \mathrm{Ni}(2+)$ transporting ATPases, CtpD and CtpJ, in Mycobacterium tuberculosis virulence. Mol Microbiol 91, 185-197, doi:10.1111/mmi.12454 (2014).

27 Lew, J. M., Kapopoulou, A., Jones, L. M. \& Cole, S. T. TubercuList--10 years after. Tuberculosis (Edinb) 91, 1-7, doi:S1472-9792(10)00111-3 [pii]

10.1016/j.tube.2010.09.008 (2011).

28 Patel, S. J. et al. Fine-tuning of Substrate Affinity Leads to Alternative Roles of Mycobacterium tuberculosis Fe2+-ATPases. J Biol Chem 291, 11529-11539, doi:10.1074/jbc.M116.718239 (2016).

29 Bulatovic, V. M. et al. Oxidative stress increases susceptibility of Mycobacterium tuberculosis to isoniazid. Antimicrob Agents Chemother 46, 2765-2771 (2002).

30 Kohanski, M. A., Dwyer, D. J., Hayete, B., Lawrence, C. A. \& Collins, J. J. A common mechanism of cellular death induced by bactericidal antibiotics. Cell 130, 797-810, doi:10.1016/j.cell.2007.06.049 (2007).

31 Ehrt, S. et al. Controlling gene expression in mycobacteria with anhydrotetracycline and Tet repressor. Nucleic Acids Res 33, e21, doi:10.1093/nar/gni013 (2005). 
41132 Ehrt, S. \& Schnappinger, D. Controlling gene expression in mycobacteria. Future

412

413

414

415

416

417

418

419

420

421

422

423

424

425

426

Microbiol 1, 177-184, doi:10.2217/17460913.1.2.177 (2006).

33 Guo, M. et al. Dissecting transcription regulatory pathways through a new bacterial onehybrid reporter system. Genome Res 19, 1301-1308, doi:10.1101/gr.086595.108 (2009).

34 Klotzsche, M., Ehrt, S. \& Schnappinger, D. Improved tetracycline repressors for gene silencing in mycobacteria. Nucleic Acids Res 37, 1778-1788, doi:10.1093/nar/gkp015 (2009).

35 BEI Resources Strain Depository, <http://www.beiresources.org> (

36 Langmead, B. \& Salzberg, S. L. Fast gapped-read alignment with Bowtie 2. Nat Methods 9, 357-359, doi:10.1038/nmeth.1923 (2012).

37 Li, H. et al. The Sequence Alignment/Map format and SAMtools. Bioinformatics 25, 2078-2079, doi:10.1093/bioinformatics/btp352 (2009).

38 Garcia, B. J., Datta, G., Davidson, R. M. \& Strong, M. MycoBASE: expanding the functional annotation coverage of mycobacterial genomes. BMC Genomics 16, 1102, doi:10.1186/s12864-015-2311-9 (2015).

39 Mi, H. et al. PANTHER version 11: expanded annotation data from Gene Ontology and Reactome pathways, and data analysis tool enhancements. Nucleic Acids Res 45, D183D189, doi:10.1093/nar/gkw1138 (2017).

\section{Acknowledgments:}

We gratefully acknowledge Jessica Winkler, Jenny Lohmiller, and Reiling Liao for their technical assistance, and we also thank Kyu Rhee and Susan Shen for helpful discussions. 
434 This work was supported by the National Institutes of Health [grant numbers U 19

$435 \quad$ Al106761; U19 Al111276; U19 Al135976; 5T32AI007509].

\section{Author Contributions:}

437 S.M. conceived of the study, led the design, generated data, analyzed the results, and

438 drafted the manuscript. B.M. developed the software to convert raw sequencing data

439 into abundance values for each TFI strain. S.H. generated data and assembled the

440 pooled TFI library cultures. J.F. assisted with sample preparation for sequencing. T.R.

441 and D.S. conceived of the study, led the design, organized the data analysis, and

442 drafted the manuscript.

443 Competing Interests:

444 The authors declare no competing interests.

445 Materials \& Correspondence:

446 Correspondence and material requests should be addressed to: David Sherman (email:

447 david.sherman@cidresearch.org). 


\section{Supplementary Information:}

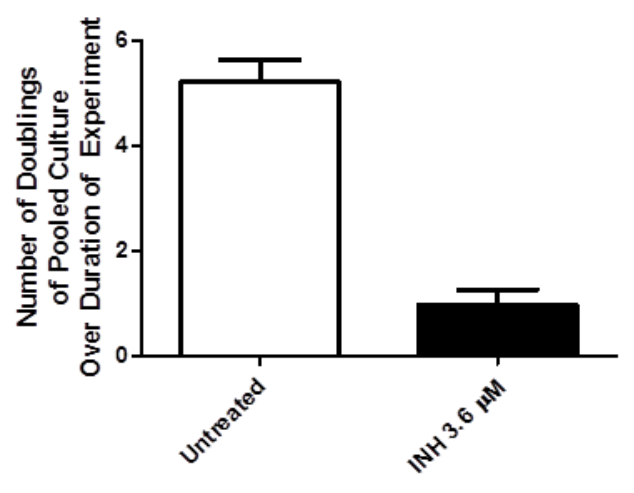

450 Figure S1: Comparing TFI pool growth between experimental conditions. Number

451 of doublings for TFI strain pool over duration of TRIP experiments in the untreated vs.

452 INH treated conditions, estimated from the change of OD600 over the course of the

453 experiment.

454 


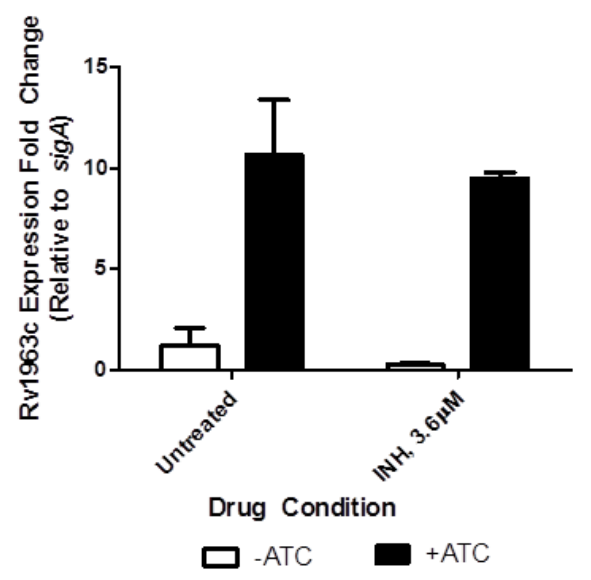

456 Figure S2: Chemical induction triggers mce3R expression change. Expression fold

457 change of Rv1963c relative to the housekeeping gene sigA, assessed by qPCR,

458 representing 3 biological replicates from a representative experiment (two were

459 performed in total). Conditions compared are in absence (white bars) and presence

460 (black bars) of anhydrous-tetracycline (ATc) inducer, and presence and absence of INH

461 exposure. Results show at least 8-fold activation of Rv1963c expression upon induction

462 with ATC in both absence and presence of INH ( $p<0.05$, Student's t-test). 


\section{A}

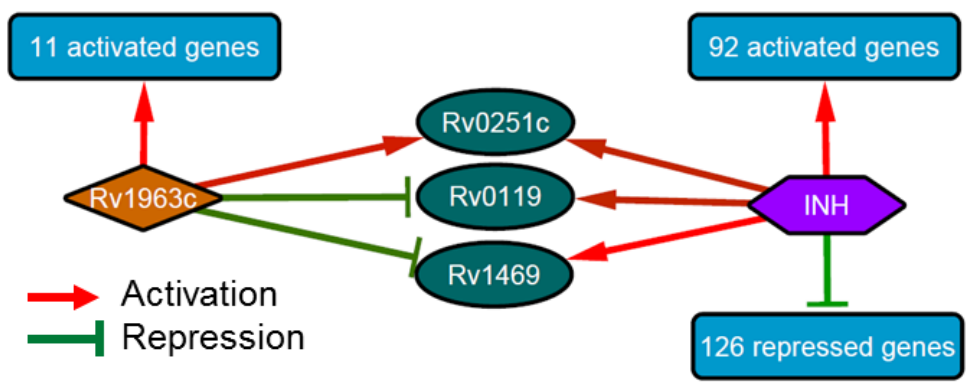

B

Figure S3: Overlap of genes regulated by $m c e 3 R$ that also modulate expression in

$470 \quad$ induction and INH exposure. 
Table S1: (See TableS1.xlsx). Detailed results of TRIP screens in presence and

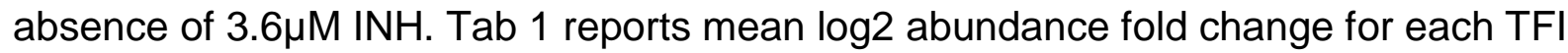
strain, relative to no induction. Tab 2 reports the log2 RPM values for each sample analyzed for the study.

Table S2: (See TableS2.xlsx). Detailed regulon and accompanying GO term enrichment information of TFs that convey altered fitness under different conditions. GO annotations were from ${ }^{38}$. Tab 1 provides information for TFs conveying growth phenotype under log-phase growth conditions. Tab 2 provides information for TFs conveying conditional resistance to INH (Group A). Tab 3 provides information for TFs conveying hypersusceptibility to INH but no growth defect in absence of drug (Group B). Tab 4 provides information for TFs conveying hypersusceptibility to INH and growth defect in absence of drug (Group C). Tab 5 provides information for all TFs conveying hypersusceptibility to INH (Groups B and C combined). The regulon information are based on data generated from ${ }^{13,14}$. The essentiality information is based on data generated from ${ }^{7,16}$.

Table S3: (See TableS3.xIsx). Overlap of TFI regulons with INH growth phenotype and genes with INH fitness phenotype in Tn-seq assay measured by ${ }^{16}$. 
Table S4: Correlation between Rv1469 regulation and INH response phenotype.

Summary of regulatory impact and TRIP abundance fold change upon INH exposure for the TFs that regulate Rv1469. Note that Rv1990c and Rv0022c do not have TRIP values associated because the raw sequence data had poor detection of these TFI strains under all conditions. Expression fold change values were assembled from ${ }^{14}$.

\begin{tabular}{|ccccc|}
\hline TF & Gene & Total & Rv1469 Expression Fold & TRIP Abundance \\
& Name & Genes & Change upon TF & Fold Change, INH \\
Rv3852 & hns & 7 & 1.05 & 0.42 \\
\hline Rv1963c & mce3R & 14 & -1.09 & -1.48 \\
\hline Rv1221 & sigE & 25 & -1.10 & -0.28 \\
\hline Rv1049 & mosR & 74 & -1.54 & -0.29 \\
\hline Rv1990c & & 91 & -1.27 & NA \\
\hline Rv0757 & phoP & 139 & -1.08 & -0.32 \\
\hline Rv0324 & & 254 & -1.21 & -0.67 \\
\hline Rv0238 & & 353 & -1.09 & 0.09 \\
\hline Rv3223c & sigH & 359 & -1.77 & -0.53 \\
\hline Rv3416 & whiB3 & 371 & -1.96 & -0.64 \\
\hline Rv0022c & whiB5 & 435 & -1.82 & NA \\
\hline
\end{tabular}

506 


\section{Table S5: Correlation between Rv0119 regulation and INH response phenotype.}

513 Summary of regulatory impact and TRIP abundance fold change upon INH exposure for

514 the TFs that regulate Rv0119, the other gene induced during basal INH exposure in

515 H37Rv and repressed by mce3R.

\begin{tabular}{|cccccc|}
\hline TF & Gene & Total & Rv0119 Expression & TRIP Abundance \\
& Name & Genes & Fold Change upon TF & Fold Change, INH \\
& & Regulated & induction (No Drug) & \\
\hline Rv0117 & oxyS & 4 & -1.58 & -0.11 \\
\hline Rv3055 & & 6 & -1.24 & 0.07 \\
\hline Rv1963c & mce3R & 14 & -1.14 & -1.48 \\
\hline Rv0260c & & 235 & -1.43 & 0.04 \\
\hline Rv0324 & & 254 & 1.03 & -0.67 \\
\hline Rv3223c & sigH & 359 & 1.28 & -0.53 \\
\hline Rv0081 & & 402 & 1.95 & -1.05 \\
\hline Rv0023 & & 893 & 1.17 & \\
\hline
\end{tabular}

